\title{
Mutation of the Signal Peptide-encoding Region of the Preproparathyroid Hormone Gene in Familial Isolated Hypoparathyroidism
}

\author{
Andrew Arnold," Susan A. Horst, “ Thomas J. Gardella, ${ }^{*}$ Hisamitsu Baba, ${ }^{*}$ Michael A. Levine, ${ }^{\star}$ and Henry M. Kronenberg* \\ *Endocrine Unit, Massachusetts General Hospital and Harvard Medical School, Boston, Massachusetts 02114; \\ ${ }^{\ddagger}$ Division of Endocrinology and Metabolism, The Johns Hopkins Medical School, Baltimore, Maryland 21205
}

\begin{abstract}
Preproparathyroid hormone (preproPTH) gene mutation has been proposed as a cause of familial isolated hypoparathyroidism (FIH). We cloned the preproPTH alleles of a patient with autosomal dominant FIH and sequenced the coding regions, $5^{\prime}$ flanking regions, and splice junctions. The putatively abnormal (based on previous linkage studies) allele differed from the other allele's normal sequence at only one nucleotide. This $T$ to $C$ point mutation changes the codon for position 18 of the 31 amino acid prepro sequence from cysteine to arginine, disrupting the hydrophobic core of the signal sequence. Because the hydrophobic core is required by secreted proteins for efficient translocation across the endoplasmic reticulum, the mutant protein is likely to be inefficiently processed. Indeed, in vitro studies demonstrated dramatically impaired processing of the mutant preproPTH to proPTH.

In summary, we observed a point mutation in the signal peptide-encoding region of a preproPTH gene in one FIH kindred and demonstrated a functional defect caused by the mutation. Mutation of the signal sequence constitutes a novel pathophysiologic mechanism in man, and further study may yield important insights both into this form of hormone deficiency and into the role of signal sequences in human physiology. (J. Clin. Invest. 1990. 86:1084-1087.) Key words: leader • PTH • point mutation
\end{abstract}

\section{Introduction}

Idiopathic hypoparathyroidism may be sporadic or familial, and may occur as an isolated defect or as a component of a more widespread disorder, such as autoimmune polyglandular failure type I or various developmental abnormalities (1). Familial isolated hypoparathyroidism $(\mathrm{FIH})^{1}$ is itself likely to comprise a heterogeneous group of disorders, with differing Mendelian modes of inheritance $(2,3)$ and distinct pathophysiological bases. Possible explanations for PTH deficiency in FIH include mutation of the preproparathyroid hormone

Address reprint requests to Dr. Andrew Arnold, Endocrine Unit, Wellman 503, Massachusetts General Hospital, Boston, MA 02114.

Dr. Baba's current address is Third Division of Internal Medicine, Kobe University School of Medicine, Kobe, Japan.

Received for publication 26 March 1990 and in revised form 29 May 1990.

1. Abbreviations used in this paper: $\mathrm{FIH}$, familial isolated hypoparathyroidism; preproPTH, preproparathyroid hormone.

J. Clin. Invest.

(c) The American Society for Clinical Investigation, Inc.

$0021-9738 / 90 / 10 / 1084 / 04 \$ 2.00$

Volume 86, October 1990, 1084-1087
(preproPTH) gene region itself, or mutations of currently undefined loci that affect the development, histologic composition, or regulation of the parathyroid glands.

In a previous study, preproPTH gene mutation was excluded as the cause of FIH in four of eight families on the basis of genetic linkage analysis using DNA polymorphisms within the gene (4). In two families, however, inheritance of a particular preproPTH allele was concordant with the presence of hypoparathyroidism. Moreover, in one family, for which inheritance of FIH was consistent with autosomal dominant transmission, the likelihood of true linkage was substantial (LOD score 1.505). Because restriction endonuclease analysis of the family's preproPTH genes failed to disclose major structural rearrangement, deletions, or insertions (4), we cloned and sequenced the putatively abnormal preproPTH gene from an affected member of this family in order to elucidate an underlying molecular defect. We found a point mutation in the signal peptide-encoding portion of this gene and have demonstrated that this mutation is associated with defective signal sequence function in vitro.

\section{Methods}

Patient II-1, a member of family D (reference 4) presented in infancy with hypocalcemic seizures, and treatment with vitamin $D$ and calcium has subsequently maintained her serum calcium levels between 1.8 and $2.1 \mathrm{mmol} /$ liter (normal range 2.1-2.6). When last evaluated at age $20 \mathrm{yr}$, serum calcium was $1.8 \mathrm{mmol} / \mathrm{liter}$, phosphate $1.5 \mathrm{mmol} /$ liter (normal 0.8-1.5), and serum PTH was $0.46 \mathrm{ng} / \mathrm{ml}$ in a midmolecule specific radioimmunoassay (normal range $0.5-1.6$ ). Serum alkaline phosphatase was $67 \mathrm{U} /$ liter (normal 50-167), serum creatinine was $67 \mu \mathrm{mol} /$ liter (normal 40-105). Renal ultrasound revealed persistent nephrocalcinosis. Her height was $157 \mathrm{~cm}$ (10-25th percentile). Chvostek sign was positive; the physical examination was otherwise normal. Hypoparathyroidism was diagnosed in her brother (patient II-5) when he was evaluated at age 1 mo for irritability, episodes of arching the back, and a hypocalcemic seizure. Treatment with vitamin $D$ and calcium has subsequently maintained his serum calcium levels between 1.9 and $2.2 \mathrm{mmol} /$ liter, although he had one episode of tetany at age 3 yr. His serum PTH level was also low but detectable $(0.48$ $\mathrm{ng} / \mathrm{ml}$ ). At age $7 \mathrm{yr}$ his height was $122.3 \mathrm{~cm}$ (50th percentile), calcium was $2.0 \mathrm{mmol} /$ liter, phosphate $1.8 \mathrm{mmol} /$ liter, alkaline phosphatase was $165 \mathrm{U} / \mathrm{liter}$, and renal function was normal.

The patient's four other siblings (II-2, II-3, II-4, and II-6) are clinically and biochemically unaffected, as is her mother (I-2). Her father (patient I-1), also healthy, had no history of hypocalcemic symptoms nor was he aware of having had symptoms in infancy consistent with hypocalcemia. His serum calcium levels were normal when evaluated in adulthood, although serum PTH levels were low-normal at 0.59 $\mathrm{ng} / \mathrm{ml}$. When hypocalcemia was induced by an intravenous infusion of EDTA he showed frankly subnormal serum PTH (Segre, G., personal communication) and urinary cAMP responses. In contrast, I-2 (mother) had normal responses to EDTA-induced hypocalcemia. No family member had evidence of mucocutaneous candidiasis, autoim- 
mune endocrine disease, or somatic features consistent with a developmental or embryological disorder.

High molecular weight genomic DNA was extracted from affected patient II-1's blood leukocytes and digested to completion with Bam $\mathrm{HI}$; a genomic library was then constructed in bacteriophage EMBL3 (5). $5 \times 10^{5}$ phage were screened by hybridization with a radioactive PTH genomic probe (" 3 ' PTH probe" in reference 6); seven independent clones were positive and were plaque purified. All of these clones contained an insert of the size $(16 \mathrm{~kb})$ expected for the preproPTH gene (6). These clones were then assessed for the presence or absence of the polymorphic Pst I restriction site, which had been used to identify the putatively abnormal and the putatively normal preproPTH alleles in this family (4). Four clones were positive for the polymorphic Pst I site, and thus contained the putatively abnormal allele, while three clones were negative for the polymorphic Pst I site and thus contained the putatively normal allele. Smaller genomic fragments $(6.3 \mathrm{~kb})$ containing the entire preproPTH gene from two independent putatively abnormal clones and one putatively normal clone were subcloned in plasmid pUC-18; appropriate subfragments of these inserts were purified and cloned into M13mp18 and M13mp19, and subjected to sequencing by the dideoxy method $(7,8)$. DNA selected for sequencing included exon I (the 5' untranslated region), the entire amino acidcoding region of preproPTH, as well as 562 bp of $5^{\prime}$ flanking region and all splice donor and acceptor sites. Both DNA strands were sequenced.

Genomic DNA specimens from affected patient I-1 and unaffected family members I-2, II-2, II-3, II-4, and II-6 were subjected to the polymerase chain reaction (9) using primers flanking preproPTH exon II. Direct DNA sequencing of the amplified fragment was then performed (10), in order to determine the presence or absence of the specific point mutation described in Results.

The plasmid pCDM-PTH-1 (kindly provided by Dr. Mason Freeman, Endocrine Unit, Massachusetts General Hospital) contains the Dde I-Xba I fragment of human preproPTH cDNA (11), inserted within the Hind III-Xba I cloning sites of the expression vector pCDM8 (12). A single base mutation (T to $C$ ), described in Results, was introduced into codon 18 of the preproPTH gene using oligonucleotide-directed mutagenesis $(13,14)$. Sequence analysis of two independent plasmids obtained from this procedure, pCDM-PTH-190 and pCDM-PTH-191, confirmed the intended T to $C$ transition; no other mutations were present.

To perform in vitro translation studies, plasmids pCDM-PTH-1 and pCDM-PTH-190 were linearized with Xba I and a sense RNA strand was transcribed using T7 RNA polymerase. The synthetic RNA was translated in a rabbit reticulocyte lysate translation system (Y4041; Promega Biotec, Madison, WI) (15), using [ $\left.{ }^{3} \mathrm{H}\right]$ leucine, with or without the addition of canine pancreatic microsomal membranes (Promega) to assess processing of the precursor peptide. Translation products were immunoprecipitated using goat anti-human PTH (1-34) antisera followed by affinity purified rabbit anti-goat IgG (0606-0082; Cappel Biomedical, Inc., Cochranville, PA) and IgGsorb (IGSL10; Enzyme Center, Inc., Malden, MA), and analyzed by SDS-polyacrylamide gel electrophoresis (16). After autoradiography of the EN $^{3}$ HANCE (NEF-981; Dupont Co., Wilmington, DE)-treated dried gel, band intensities were quantitated by densitometry. Maximal processing was observed with the addition of $4 \mu \mathrm{l}(8 \mathrm{EQ})$ of membranes (range 2-6 $\mu$ l tested).

\section{Results}

The nucleotide sequence of the $5^{\prime}$ flanking, coding, and splice junction regions of the affected patient's presumedly normal preproPTH gene (marked by the absence of the polymorphic Pst I site) was indeed normal. The corresponding sequences of the putatively abnormal allele differed from the normal sequence at only a single nucleotide (Fig. 1). This $\mathrm{T}$ to $\mathrm{C}$ transition in exon II results in the substitution of arginine (CGT) for cysteine (TGT) at position 18 in the 25 amino acid signal or

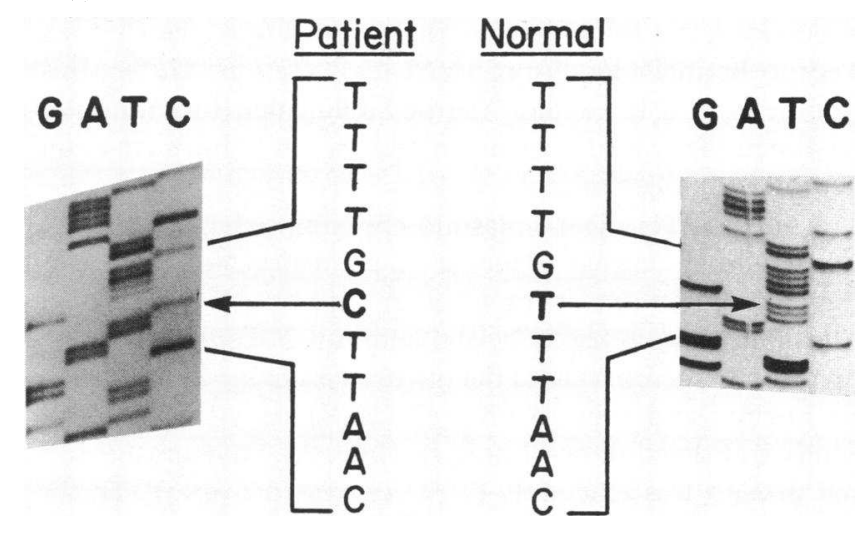

Figure 1. Demonstration of patient's preproPTH gene point mutation. Section of the sequencing gel autoradiogram for the patient's abnormal preproPTH allele (left), compared with the normal preproPTH sequence (from the patient's other allele) in the same region of exon II (right). The nucleotide involved in the thymidine to cytosine point mutation is shown in bold type, with arrows pointing to the corresponding bands on the sequencing gels. The normal nucleotide sequence shown is identical to that previously described (17).

proPTH was unaltered. The mutation was confirmed by identification of the corresponding A to $G$ transition on this clone's complementary strand of DNA, and also by sequencing, with the same result, the putatively abnormal preproPTH gene from a completely independent primary phage clone.

Clinical and genetic linkage studies predicted that the patient's father would also carry the above mutation on one preproPTH allele. This hypothesis was indeed confirmed by direct nucleotide sequencing after amplification of exon II of his preproPTH genes using the polymerase chain reaction. In addition, the family members predicted to have normal preproPTH alleles did not carry this mutation (data not shown). Confirmation of the mutation's presence in affected sibling II-5 was not possible due to unavailability of DNA.

The exon II $\mathrm{T}$ to $\mathrm{C}$ point mutation introduces a charged amino acid in the midst of the hydrophobic core of preproPTH's signal sequence (Fig. 2). Because the hydrophobic core is crucial for translocation of proteins destined for secretion across the membranes of the endoplasmic reticulum (see

ATG aTA CCT GCA AAA GaC ATG GCT AAA GTT ATG ATT GTC ATG TTG GCA ATT
Het Ile Pro Ala Lys Asp met Ala Lys Val Net Ile val Met leu Ala Ile $\begin{array}{lllllllllllllllll}1 & 2 & 3 & 4 & 5 & 6 & 7 & 8 & 9 & 10 & 11 & 12 & 13 & 14 & 15 & 16 & 17\end{array}$

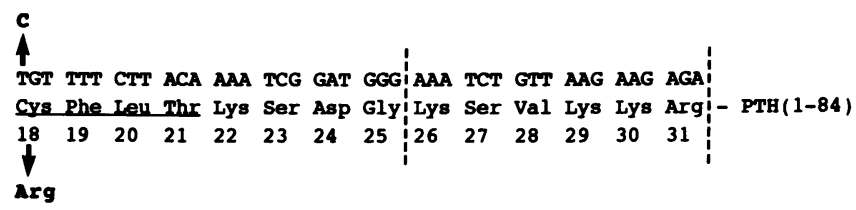

Figure 2. Nucleotide and amino acid sequences of the signal "pre" and "pro" regions of preproPTH. Amino acids 1-25 comprise the signal peptide; residues $26-31$ constitute the pro sequence, and the 84 amino acids of mature PTH follow (11). Vertical broken lines indicate the respective cleavage sites. Amino acids (10-21), comprising the hydrophobic core of the signal sequence, are underlined. The described patient's mutation at position 18 is indicated by arrows and bold type. 
Discussion), we hypothesized that the mutant protein was likely to be inefficiently processed for secretion. To test this hypothesis, and to exclude the possibility that the mutation was functionally silent, we examined the mutation's effect on preproPTH processing.

A preproPTH cDNA plasmid containing the $\mathrm{T}$ to $\mathrm{C}$ substitution in the signal sequence was constructed, RNA was transcribed, and processing of its in vitro translation product to proPTH was assessed by the addition of canine pancreas microsomal membranes to the in vitro lysate. Fig. 3 shows that the normal and mutant preproPTH RNAs were both efficiently translated (lanes 1 and 3). However, whereas the normal protein was efficiently (75\%) processed to proPTH, the abnormal preproPTH protein was poorly $(4.7 \%)$ processed (lanes 2 and 4). These results strongly suggest that the 18 (Cys $\rightarrow$ Arg) preproPTH signal sequence mutation indeed adversely alters the manner in which the protein interacts with the processing pathway.

\section{Discussion}

We have described a point mutation in the signal peptide-encoding region of the preproPTH gene in an FIH kindred. This mutation is likely to be the primary defect that leads to hypoparathyroidism in affected members of this family because $(a)$ previous RFLP studies indicated that inheritance of PTH deficiency in this family was tightly linked to inheritance of the particular preproPTH allele shown here to contain the mutation, and $(b)$ when this mutation (and only this mutation) was introduced into normal preproPTH cDNA and expressed in vitro, dramatically impaired processing of the mutant protein was observed.

Defective signal sequences have not previously been implicated as the cause of human disease. Signal sequences are present within the precursors of most secreted proteins, and are required to direct these proteins into the cell's secretory pathway. Signal recognition particles bind signal sequences and then bind to receptors (docking proteins) in the microsomal membrane, thus delivering the precursor protein to the outer border of the endoplasmic reticulum. The precursor protein is then inserted into the membrane of the endoplasmic reticulum and translocated; the signal sequence is cleaved as the protein completes its passage across the membrane. The signal sequence may thereby interact with a series of different proteins during these discrete early steps of delivery, translo-

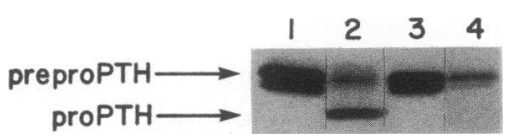

Figure 3. Autoradiogram of $\left[{ }^{3} \mathrm{H}\right]$ leucinelabeled proteins derived from the translation of RNA transcribed from

plasmids containing normal (lanes 1 and 2) and mutant (lanes 3 and 4) preproPTH cDNA, i.e., plasmids pCDM-PTH-1 and pCDMPTH-190, respectively. Lanes 1 and 3 show translated immunoprecipitated protein in the absence of added microsomal membranes, while lanes 2 and 4 show translation (and processing to proPTH) in the presence of microsomal membranes. Arrows indicate the positions of the bands representing preproPTH (top) and proPTH (bottom). PreproPTH is represented by a closely spaced doublet because it consists of both preproPTH and a truncated protein beginning at Met-7 of preproPTH. The truncated precursor functions normally in vitro and in intact cells (18). cation, and cleavage (reviewed in 19). Subsequent steps, including export to the Golgi and actual secretion, occur after signal cleavage.

The structural requirements of functional signal sequences are imprecisely characterized, but one universal characteristic is the presence of a stretch of uncharged, predominantly hydrophobic amino acids. The introduction of charged residues into the hydrophobic region of prokaryotic signal sequences often dramatically interferes with signal function (19). Analogous genetic studies in eukaryotic systems are less extensive, but nevertheless support the important role of the hydrophobic core (20). The preproPTH 18 (Cys $\rightarrow$ Arg) mutation may exert its dramatic effect on signal function by introducing a positive charge at position 18 specifically, or by shortening the uninterrupted hydrophobic stretch to only eight contiguous amino acids. Alternatively, the loss of cysteine- 18 might be functionally important; this is unlikely, since Cioffi et al. (21) showed that deleting this cysteine or substituting leucine for cysteine in the closely homologous bovine preproPTH had no deleterious effect on processing. The data in Fig. 3, as well as the presence of PTH deficiency in the affected patients, support our hypothesis that the defect impairs release of PTH molecules from the parathyroid gland. The mutant gene described here thus provides a model for a new class of functionally important mutations in human disease; signal mutations might be present in precursors of other secreted proteins and cause analogous genetic deficiencies.

This example of human genetic disease can be considered together with several other conditions affecting the secretory pathway. For example, in alpha-1-antitrypsin deficiency, one mutation in the body of the mature protein (Z-variant) results in aggregation and impaired export of the protein from the endoplasmic reticulum to the Golgi apparatus (22). Additionally, specific mutations in the connecting segment region of the proinsulin gene cause abnormal cleavage of the prohormone in the trans-Golgi network, resulting in hyperproinsulinemia (23). In contrast to these abnormalities associated with late stages of the secretory pathway, the signal sequence mutation in the patient reported here leads to a defect in an early stage, the translocation of the precursor across the endoplasmic reticular membrane.

Although the size of the family studied here is small, inheritance of hypoparathyroidism is most consistent with an autosomal dominant pattern (see Methods and reference 4). Our observation that the coding sequence of the patient's second preproPTH gene was normal is consistent in that regard. The apparent inability of the normal preproPTH allele to yield sufficient secreted PTH to prevent hypocalcemia poses an interesting pathophysiologic question. One possible explanation is that the affected patient's remaining preproPTH allele, in spite of its normal coding and near 5 ' regions, is actually abnormal, e.g., in an unknown regulatory element, and therefore not well expressed. While certainly not excluded by our data, this possibility is unlikely in that the two affected siblings (II-1 and II-5) each inherited different preproPTH alleles from their normal mother (4). Another hypothetical explanation for this dominance is that the mutant protein may successfully bind to the membrane, but may not be able to move forward through the translocation process. Mutant protein could thereby obstruct this pathway, and cause a global defect in protein processing in parathyroid cells that would affect the normal preproPTH product and possibly other unrelated secreted and 
membrane proteins as well. Analogous dominant mutations have been described in Escherichia coli (24). Future studies will be directed toward testing this hypothesis, as well as toward determining the precise stage in the processing pathway that is affected by this mutation.

The signal sequence defect does not completely abolish release of PTH since, even in the hypocalcemic children, PTH levels, while low, were still detectable. Their affected father appears further on a spectrum of improved ability to secrete PTH, since he could achieve normocalcemia despite low-normal PTH levels and impaired secretory reserve. The ultimate clinical expression of a single gene defect is often determined by additional genetic, physiologic, or regulatory factors. Such modulating influences might account for the difference in clinical severity that the PTH processing defect produces in the affected children as compared with the father in this family. It will be interesting to observe, for example, whether the severity of hypoparathyroidism lessens as the affected children become older, since an age-dependent influence on expression of genetic hypoparathyroidism has been described (3); persistent hypoparathyroidism in the older child, however, argues against an important role for such an influence. Alternatively, the mildly but definitely affected father might possess a variant allele of another gene that partially improves preproPTH translocation; such a variant could permit a membrane protein in the translocation pathway to interact more successfully with the mutant preproPTH. Thus, further insight into these different clinical expressions of the preproPTH mutation will likely require a better definition of the components of the translocation pathway.

In summary, a preproPTH gene from an FIH kindred contains a functionally significant point mutation in its signal peptide-encoding region. Because signal sequence mutation has not previously been implicated in human disease, study of this family and gene may yield important insights both into this form of hormone deficiency and into the role of signal sequences in human physiology.

\section{Acknowledgments}

We are grateful to Drs. David E. C. Cole, Donald Fraser, Richard B. Goldbloom, and Sang Whay Koo for their assistance in providing clinical information and patient samples for these studies. We wish to thank Mrs. Madge Hypolite for expert preparation of the manuscript.

Supported by grants from the National Institutes of Health (DK-11794, DK-38990). Dr. Arnold is the recipient of a Junior Faculty Research Award from the American Cancer Society.

\section{References}

1. Aurbach, G. D., S. J. Marx, and A. M. Spiegel. 1985. Parathyroid hormone, calcitonin, and the calciferols. In Williams Textbook of Endocrinology. 7th ed. J. D. Wilson and D. W. Foster, editors. W. B. Saunders Co., Philadelphia, PA. 1137-1217.

2. Whyte, M. P., and V. V. Weldon. 1981. Idiopathic hypoparathyroidism presenting with seizures during infancy: $X$-linked recessive inheritance in a large Missouri kindred. J. Pediatr. 99:608-611.

3. Winter, W. E., J. H. Silverstein, N. K. Maclaren, W. J. Riley, and J. J. Chiaro. 1983. Autosomal dominant hypoparathyroidism with variable, age-dependent severity. J. Pediatr. 103:387-390.

4. Ahn, T. G., S. E. Antonarakis, H. M. Kronenberg, T. Igarashi, and M. A. Levine. 1986. Familial isolated hypoparathyroidism: a molecular genetic analysis of 8 families with 23 affected persons. Medicine (Baltimore). 65:73-81.
5. Karn, J., and S. Brenner. 1983. New bacteriophage lambda vectors with positive selection for cloned inserts. Methods Enzymol. 101:3-19.

6. Arnold, A., H. G. Kim, R. D. Gaz, R. L. Eddy, Y. Fukushima, M. G. Byers, T. B. Shows, and H. M. Kronenberg. 1989. Molecular cloning and chromosomal mapping of DNA rearranged with the parathyroid hormone gene in a parathyroid adenoma. J. Clin. Invest. 83:2034-2040.

7. Messing, J. 1983. New M13 vector for cloning. Methods Enzymol. 101:20-78.

8. Sanger, F., S. Nicklen, and A. R. Coulson. 1977. DNA sequencing with chain terminating inhibitors. Proc. Natl. Acad. Sci. USA. 74:5463-5467.

9. Saiki, R. K., D. H. Gelfand, S. Stoffel, S. J. Scharf, R. Higuchi, G. T. Horn, K. B. Mullis, and H. A. Erlich. 1988. Primer-directed enzymatic amplification of DNA with a thermostable DNA polymerse. Science (Wash. DC). 239:487-491.

10. Wong, C., C. E. Dowling, R. K. Saiki, R. G. Higuchi, H. A. Erlich, and H. H. Kazazian, Jr. 1987. Characterization of $\beta$-thalassemia mutations using direct genomic sequencing of amplified single copy DNA. Nature (Lond.). 330:384-386.

11. Hendy, G. N., H. M. Kronenberg, J. T. Potts, Jr., and A. Rich. 1981. Nucleotide sequence of cloned cDNAs encoding human preparathyroid hormone. Proc. Natl. Acad. Sci. USA. 78:7365-7369.

12. Seed, B. 1987. An LFA-3 cDNA encodes a phospholipid-linked membrane protein homologous to its receptor CD2. Nature (Lond.). 329:840-842.

13. Zoller, M. J., and M. Smith. 1983. Oligonucleotide-directed mutagenesis of DNA fragments cloned into M13 vectors. Methods Enzymol. 100:468-500.

14. Taylor, J. W., W. Schmidt, R. Cosstick, A. Okruszek, and F. Eckstein. 1985. The use of phosphorothioate-modified DNA in restriction enzyme reactions to prepare nicked DNA. Nucleic Acids Res. 24:8749-8785.

15. Pelham, H. R. B., and R. J. Jackson. 1976. An efficient mRNA-dependent translation system from reticulocyte lysates. Eur. J. Biochem. 67:247-256.

16. Laemmli, U. K. 1970. Cleavage of structural proteins during the assembly of the head of bacteriophage T4. Nature (Lond.). 227:680-685.

17. Vasicek, T. J., B. E. McDevitt, M. W. Freeman, B. J. Fennick, G. N. Hendy, J. T. Potts, Jr., A. Rich, and H. M. Kronenberg. 1983. Nucleotide sequence of the human parathyroid hormone gene. Proc. Natl. Acad. Sci. USA. 80:2127-2131.

18. Freeman, M. W., K. M. Wiren, A. Rapoport, M. Lazar, J. T. Potts, Jr., and H. M. Kronenberg. 1987. Consequences of amino-terminal deletions of preproparathyroid hormone signal sequence. Mol. Endocrinol. 1:628-638.

19. Emr, S. D., M. N. Hall, and T. J. Silhavy. 1980. A mechanism of protein localization: the signal hypothesis and bacteria. J. Cell Biol. 86:701-711.

20. Kaiser, C. A., and D. Botstein. 1986. Secretion-defective mutations in the signal sequence for Saccharomyces cerevisiae invertase. Mol. Cell. Biol. 6:2382-2391.

21. Cioffi, J. A., K. L. Allen, M. O. Lively, and B. Kemper. 1989. Parallel effects of signal peptide hydrophobic core modifications on co-translational translocation and post-translational cleavage by purified signal peptidase. J. Biol. Chem. 264:15052-15058.

22. Verbanac, K. M., and E. C. Heath. 1986. Biosynthesis, processing, and secretion of $\mathrm{M}$ and $\mathrm{Z}$ variant human $\alpha_{1}$-antitrypsin. J. Biol. Chem. 261:9979-9989.

23. Robbins, D. C., S. E. Shoelson, A. H. Rubenstein, and H. S. Tager. 1984. Familial hyperproinsulinemia: two cohorts secreting indistinguishable type II intermediates of proinsulin conversion. J. Clin. Invest. 73:714-719.

24. Hall, M. N., and T. J. Silhavy. 1981. Genetic analysis of the major outer membrane proteins of Escherichia coli. Annu. Rev. Genet. 15:91-142. 